\title{
Stabilization and relative phase effects in a dichromatically driven diatomic Morse molecule: Interpretation based on nonlinear classical dynamics
}

\author{
Vassilios Constantoudis ${ }^{\text {a) }}$ \\ Physics Department, National Technical University, Athens, Greece and Institute of Microelectronics, NCSR \\ "Demokritos" Aghia Paraskevi, 15310 Athens, Greece \\ Cleanthes A Nicolaides ${ }^{\text {b) }}$ \\ Physics Department, National Technical University, Athens, Greece and Theoretical and Physical Chemistry \\ Institute, National Hellenic Research Foundation, Athens 11635, Greece
}

(Received 20 September 2004; accepted 10 December 2004; published online 22 February 2005)

\begin{abstract}
The dissociation dynamics of a dichromatically laser-driven diatomic Morse molecule vibrating in the ground state is investigated by applying tools of the nonlinear theory of classical Hamiltonian systems. Emphasis is placed on the role of the relative phase of the two fields, $\varphi$. First, it is found that, just like in quantum mechanics, there is dependence of the dissociation probability on $\varphi$. Then, it is demonstrated that addition of the second laser leads to suppression of probability (stabilization), when the intensity of the first laser is kept constant just above or below the single laser dissociation threshold. This "chemical bond hardening" diminishes as $\varphi$ increases. These effects are investigated and interpreted in terms of modifications in phase space topology. Variations of $\varphi$ as well as of the intensity of the second laser may cause (i) appearance/disappearance of the stability island corresponding to the common resonance with the lowest energy and (ii) deformation and movement of the region of Kolmogorov-Arnold-Moser tori that survive from the undriven system. The latter is the main origin in phase space of stabilization and $\varphi$ dependence. Finally, it is shown that the use of short laser pulses enhances both effects. (C) 2005 American Institute of Physics.
\end{abstract}

[DOI: $10.1063 / 1.1854631]$

\section{INTRODUCTION}

The dynamics of atoms and molecules driven simultaneously by two-color fields of commensurate frequencies $\omega$ and $\mathrm{n} \omega, \mathrm{n}=2,3, \ldots$, is a subject of considerable interest, mainly because quantum mechanics predicts that the superposition of two fields, each with its own controlled parameters, gives rise to phenomena that single laser excitation cannot produce. The most conspicuous such phenomenon is the dependence of the processes of dissociation or ionization on the relative phase $\varphi$ of the two laser fields. ${ }^{1-3}$ The effect of $\varphi$ on the ionization/dissociation yield has been demonstrated by two-color experiments that have been conducted using either $1 \omega-2 \omega$ or $1 \omega-3 \omega$ fields, ${ }^{4-6}$ as well as by corresponding theoretical works and numerical applications. ${ }^{7-10}$ These developments have been interpreted in the context of quantum interference since the second frequency adds alternative pathways towards fragmentation, thereby adding transition amplitudes causing interference effects that are sensitive to relative phase variations.

As is well known, there is also interest in the application of nonlinear classical mechanics to quantum systems, one of the aims being to determine areas of correspondence between classical and quantum mechanics and to discover possibilities of applications where classical mechanics produces meaningful and physically relevant results.

In this context, as a continuation of our investigation of

\footnotetext{
${ }^{a)}$ Electronic mail: vconst@imel.demokritos.gr

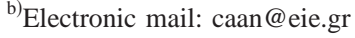

multiphoton molecular dissociation, ${ }^{11,12}$ we undertook an extensive nonlinear classical dynamics study of the dissociation dynamics of a dichromatically laser-driven diatomic Morse molecule placing emphasis on the possible effects of the phase difference $\varphi$. Our related optimism was supported by the publication of Sirko and Koch, ${ }^{6}$ who reported a combined experimental and theoretical study on the influence of the relative phase on the ionization probability of a highly excited $\mathrm{H}$ atom that is driven dichromatically by microwave fields, in which it is indicated that classical dynamics can provide an adequate interpretation of certain observations. In particular, Sirko and Koch showed that variations of $\varphi$ affect the structure of the common resonances in phase space and so can be used for the control of the ionization probability. The presence of common resonances requires rational frequency ratio ( $1 \omega-3 \omega$ in the Sirko-Koch experiment). Their existence was demonstrated for the first time in the model of driven one-dimensional hydrogen atoms by Howard, ${ }^{13}$ without, however, further investigation. The work of Ref. 6 focused on the interpretation of the specific experimental behavior and, consequently, was limited to specific choices of values for field intensity, initial energy, pulse shape, and relative phase.

It is then our conclusion that, as regards the general theory of classical dynamics, a thorough investigation of the relative phase effects on the escape dynamics, (ionization or dissociation), and of its dependence on the system and field parameters, has heretofore been absent. The work reported here aims at rectifying this situation, via an application in the 
area of molecular dissociation dynamics. In order to be general, the study has utilized the Morse potential for the description of vibrational motion, a potential that has been used before in classical and in quantum mechanical investigations of dissociation dynamics induced by a laser field. ${ }^{11,14-16}$ Along with the ionization of $\mathrm{H}$ atoms, its laser-induced dissociation behavior constitutes a paradigm for the phenomenology of the quantum mechanical manifestations of regular and chaotic behavior in classical phase space, this being the core of the quantum chaos problem.

Apart from the dissociation of Morse molecules by single frequency laser, effects of the simultaneous driving with two laser fields has also been investigated, both classically and quantum mechanically. ${ }^{14,16-18}$ The main concern in those studies was to find the field parameters for which the addition of the second laser enhances the dissociation process, thereby lowering the required field intensity for the occurence of dissociation. In phase space terms, the key idea is the following: by properly tuning the second frequency, one aims at interspersing the nonlinear resonances of the second field together with their chaotic layers between the resonances of the first laser. In this way, the overlapping of the resonances and the concomitant emergence of dissociation should take place at lower intensities than those in the single laser case. Numerical calculations and analytical arguments have been used to demonstrate this idea. However, no work has been reported yet on the effects of $\varphi$, and of its combination with variations of intensity, on the dissociation process.

In the present paper, we first demonstrate numerically that classical dynamics can indeed produce effects connected to the dependence of the dissociation probability on $\varphi$. We report results from extensive computations on the dependence of this effect on the values of laser intensities and pulse duration. We then demonstrate the main conclusion of the paper, namely, that the classical dynamics prediction of the relative phase effect is associated with a stabilization phenomenon, i.e., with suppression of the dissociation probability (chemical bond hardening) induced by the addition of the second laser. Finally, the effects of variations of $\varphi$ and of the second laser intensity on phase space structures are explored and the origins of the above-mentioned effects are determined.

\section{DESCRIPTION OF THE SYSTEM AND METHODOLOGY}

Since the aim of this work is not to reproduce experimentally determined energy levels or lifetimes, but to uncover and explore general trends in the dissociation behavior, some approximations are both legitimate and necessary for the facilitation of the calculations. The approximations include the system, the laser fields, and their interaction. First, we consider the pure vibrational motion of a diatomic molecule for a fixed electronic configuration, under the assumption that rotational motion is not involved in the dissociation process. The molecular potential is modeled by a Morse potential,

$$
V_{M}(r)=D\left(1-e^{-\alpha\left(r-r_{e}\right)}\right)^{2},
$$

where $\mathrm{D}$ is the dissociation energy, $\mathrm{r}_{\mathrm{e}}$ is the equilibrium bond distance, and $\alpha^{-1}$ is the range of the potential. HF, HCL, $\mathrm{H}_{2}$, and $\mathrm{NO}$ are some examples of diatomic molecules that have been modeled by the Morse potential.

The shape of the laser pulses is chosen to be trapezoidal. The case of ac fields with constant amplitudes during the whole interaction has also been considered. The interaction between the molecule and the lasers is chosen to be a linear dipole function. Selected calculations with other functions did not show significant modifications of the results. Thus, the Hamiltonian of the system is written as

$H(r, t)=H_{o}(r)+\left(r-r_{e}\right) \varepsilon(t)\left[A_{1} \sin \left(f_{1} t\right)+A_{2} \sin \left(f_{2} t+\varphi\right)\right]$,

where $H_{o}(r)=p^{2} / 2 m+V_{M}(r)$ is the unperturbed Hamiltonian, $A_{1}$ and $A_{2}$ are the maximum amplitudes of the electric fields of two lasers, $\varepsilon(t)$ is the envelope of the laser pulses (the same for both lasers), $f_{1}$ and $f_{2}$ are the corresponding laser frequencies, and $\varphi$ is the relative phase of the two lasers. The computational methodology involves the solution of the equations of the classical dynamics of the time-dependent nonlinear Hamiltonian (2) in conjunction with an appropriate choice of the ensemble of initial conditions.

If we introduce the dimensionless variables

$$
\begin{aligned}
& x=1-e^{-\alpha\left(r-r_{e}\right)}, \\
& y=p / \sqrt{2 D m}, \\
& \tau=\alpha \sqrt{2 D / m} t, \\
& F_{i}=A_{i} /(2 D \alpha), \quad i=1,2, \\
& \omega_{i}=f_{i} / \Omega_{\mathrm{M}}, \quad i=1,2,
\end{aligned}
$$

with $\Omega_{\mathrm{M}}=\sqrt{2 D \alpha^{2} / m}$ being the Morse harmonic frequency and $m$ the reduced mass, then Hamilton's equations become

$$
\begin{aligned}
& d x / d \tau=(1-x) y \\
& d y / d t=-(1-x) x-\varepsilon(t)\left[F_{1} \sin \left(\omega_{1} \tau\right)+F_{2} \sin \left(\omega_{2} \tau+\varphi\right)\right]
\end{aligned}
$$

Although Eq. (4) is in a form appropriate for numerical computation, the initial conditions are expressed in action-angle variables $(1, \theta)$, or, more conveniently, in $(E, \theta)$, where $E=I-I^{2} / 4$ is the dimensionless energy (energy divided by D) of the unperturbed Morse oscillator. ${ }^{19}$ For bound states 0 $<\mathrm{E}<1$ the transformation relations between $(E, \theta)$ and $(x, y)$ are given by

$$
\begin{aligned}
& x=(E+\sqrt{E}+\cos \theta) /(1+\sqrt{E} \cos \theta), \\
& y=-\Omega_{M}+\sqrt{E} \sin \theta /(1+\sqrt{E} \cos \theta) .
\end{aligned}
$$

In order to imitate more closely the quantum photodissociation process, the initial classical states is chosen as an 
TABLE I. Fixed and varied system parameters

\begin{tabular}{ll}
\hline \hline System Parameters & \\
\hline Field amplitudes & Varied \\
Field frequencies & Fixed \\
Pulse shape & Varied \\
Pulse duration & Varied \\
Initial energy & Fixed \\
Relative phase & Varied \\
\hline \hline
\end{tabular}

ensemble of initial conditions with specific energy $\mathrm{E}$ and angle variables $\theta$ uniformly distributed between $-\pi$ and $\pi .^{11,16-20}$

The dissociation dynamics of the system (2) depends on several parameters: normalized field frequencies $\left(\omega_{1}, \omega_{2}\right)$ and amplitudes $\left(F_{1}, F_{2}\right)$, pulse shape $\varepsilon(t)$ and duration, relative field phase, $\varphi$, and energy of the initial state $E$.

All calculations reported below were done keeping the frequencies $\omega_{1,2}$ of two lasers and the initial vibration energy constant. The fast laser frequency is redshifted from the Morse harmonic frequency $\Omega_{\mathrm{M}}$ and its normalized value is taken as $\omega_{2}=0.84$, so that single laser driving leads to maximal dissociation yield for all laser amplitudes. ${ }^{11,12,16,21-23}$ The frequency of the slow laser is chosen to be one third of the fast laser: $\omega_{1}=\omega_{2} / 3=0.28$. Also, the molecule is considered to vibrate initially in its ground state. For the molecular constants of $\mathrm{HF},{ }^{16,19}$ the frequency of the fast laser is $f_{2}$ $=3476 \mathrm{~cm}^{-1}$ while the normalized ground state energy is $E_{o}=0.045$. A normalized field amplitude $F_{1}$ or $F_{2}=1$ corresponds to a laser intensity of $320 \mathrm{TW} / \mathrm{cm}^{2}$. The remaining parameters are varied so as to examine their effect on the properties of the dissociation process. The main interest will be in mapping the dissociation behavior on the plane of the normalized field amplitudes $\left(F_{1}, F_{2}\right)$ and examine the regions of maximum relative phase or stabilization effects on dissociation. Table I summarizes the system parameters.

\section{RELATIVE PHASE EFFECTS ON DISSOCIATION DYNAMICS}

Before reporting the results of our calculations on the effects of $\varphi$ on the dissociation probability, we discuss the dissociation criterion for the trajectory solutions of the classical equations. To this end, we define the normalized compensated energy by

$$
E_{C}=x^{2}+\left[y-\frac{F_{1}}{\omega_{1}} \cos \left(\omega_{1} t\right)-\frac{F_{2}}{\omega_{2}} \cos \left(\omega_{2} t+\varphi\right)\right]^{2},
$$

which does not include the oscillations of the real energy that is caused by the oscillations of the time-dependent fields. According to relevant literature, e.g., Refs. 15 and 20, a molecular bond can be considered broken when $\mathrm{E}_{\mathrm{C}}$ exceeds the dissociation limit 1. For an initial classical state consisting of a number of initial conditions (in our calculations $\sim 500$ ) with a specific energy, the dissociation probability is defined as the fraction of initial conditions that lead to dissociated trajectories during the interaction with the laser pulses.

Based on this criterion and definitions, we can calculate the dependence of the dissociation probability $\mathrm{P}_{\mathrm{D}}$ of the

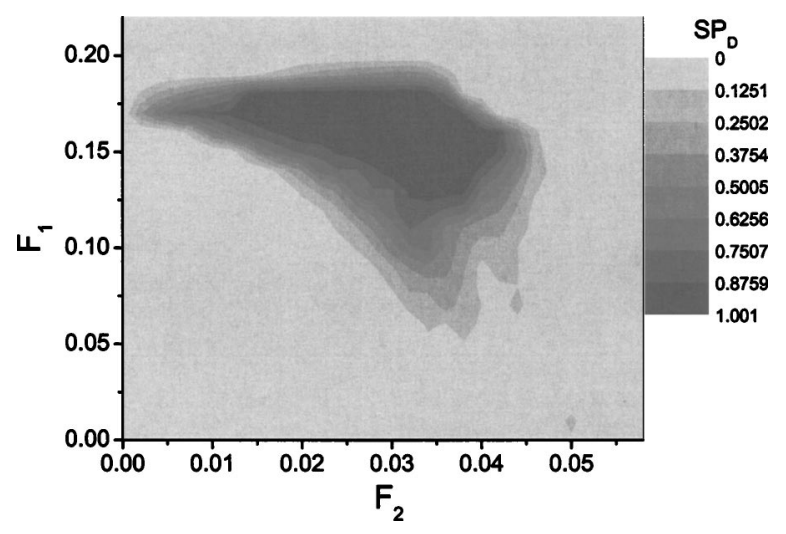

FIG. 1. Contour plot on the plane $\mathrm{F}_{2}-\mathrm{F}_{1}$ of the sensitivity $\mathrm{SP}_{\mathrm{D}}$ defined as the maximum recorded difference of $\mathrm{P}_{\mathrm{D}}$ as $\varphi$ varies (see text for details). The darker the region is the greater is the sensitivity of $\mathrm{P}_{\mathrm{D}}$ to changes of $\varphi$.

ground state $\left(E=E_{0}=0.045\right)$ on $\varphi$ for laser pulses of trapezoidal shape with total duration of 40 periods of the slow laser $(\sim 1 \mathrm{~ns})$ and rising time equal to ten periods. In order to obtain a better understanding of the influence of intensity on the control of dissociation via the relative phase, we proceed with the estimation of the sensitivity $\mathrm{SP}_{\mathrm{D}}$ of $\mathrm{P}_{\mathrm{D}}$ to $\varphi$, on a grid in the $\mathrm{F}_{2}-\mathrm{F}_{1}$ plane. At each grid point $\left(\mathrm{F}_{2}, \mathrm{~F}_{1}\right), \mathrm{SP}_{\mathrm{D}}$ is defined as

$$
\mathrm{SP}_{D}\left(F_{2}, F_{1}\right)=P_{D}^{\max }\left(F_{2}, F_{1}\right)-P_{D}^{\min }\left(F_{2}, F_{1}\right)
$$

where $P_{D}^{\max }\left(F_{2}, F_{1}\right)=\max \left[P_{D}\left(F_{2}, F_{1} ; \varphi\right), 0<\varphi \leqslant 2 \pi\right]$ and $P_{D}^{\min }\left(F_{2}, F_{1}\right)=\min \left[P_{D}\left(F_{2}, F_{1} ; \varphi\right), 0<\varphi \leqslant 2 \pi\right]$.

Taking into account that $P_{D}(\varphi)=P_{D}(2 \pi-\varphi)$, (see Ref. $24)$, we implement the above estimation by calculating $P_{D}$ for $\varphi=0^{\circ}, 30^{\circ}, 60^{\circ}, 90^{\circ}, 120^{\circ}, 150^{\circ}, 180^{\circ}$. Figure 1 shows a contour plot of $\mathrm{SP}_{D}\left(F_{2}, F_{1}\right)$ for initial energy equal to $E_{o}$. We observe that the region where $\mathrm{SP}_{D}$ comes close to its maximum value $\left(\mathrm{SP}_{D}>0.87\right)$ is clearly located above the diagonal $F_{1}=F_{2}$, i.e., the effect of the relative phase on $P_{D}$ is maximized for slow laser intensities larger than those of the fast laser $\left(F_{1}>F_{2}\right)$.

In order to see how this sensitivity to $\varphi$ variations emerges, we show in Fig. 2 some representative $P_{D}$ maps for $\varphi=0^{\circ}, 30^{\circ}, 90^{\circ}, 180^{\circ}$. They reveal that the $\varphi$-dependence effect is associated with a stabilization phenomenon (reduction of the $P_{D}$ ) that is prominently present at $\varphi=0^{\circ}$ and disappears as $\varphi$ increases. The stabilization (or chemical bond hardening) occurs in two cases. First, for slow laser amplitudes just above the single slow laser dissociation threshold $\left(F_{1}^{\text {thres }}\right.$ $\approx 0.13$ ), when the second fast laser is added (fast laser stabilization). Second, for fast laser amplitudes about the single fast laser threshold $\left(F_{2}^{\text {thres }} \approx 0.042\right)$ when the slow laser is added (slow laser stabilization).

To get an idea of the magnitude of the intensities involved in such relative phase and stabilization effects, the following association is noted. For molecular constants of the HF molecule, the normalized amplitude $F_{1}=0.18$ corresponds to laser intensity of $5.8 \times 10^{13} \mathrm{~W} / \mathrm{cm}^{2}$ while $F_{2}$ $=0.04$ corresponds to $1.3 \times 10^{13} \mathrm{~W} / \mathrm{cm}^{2}$. The fast laser dissociation starts immediately after the application of the fast laser, whereas the slow laser dissociation occurs after an ini- 


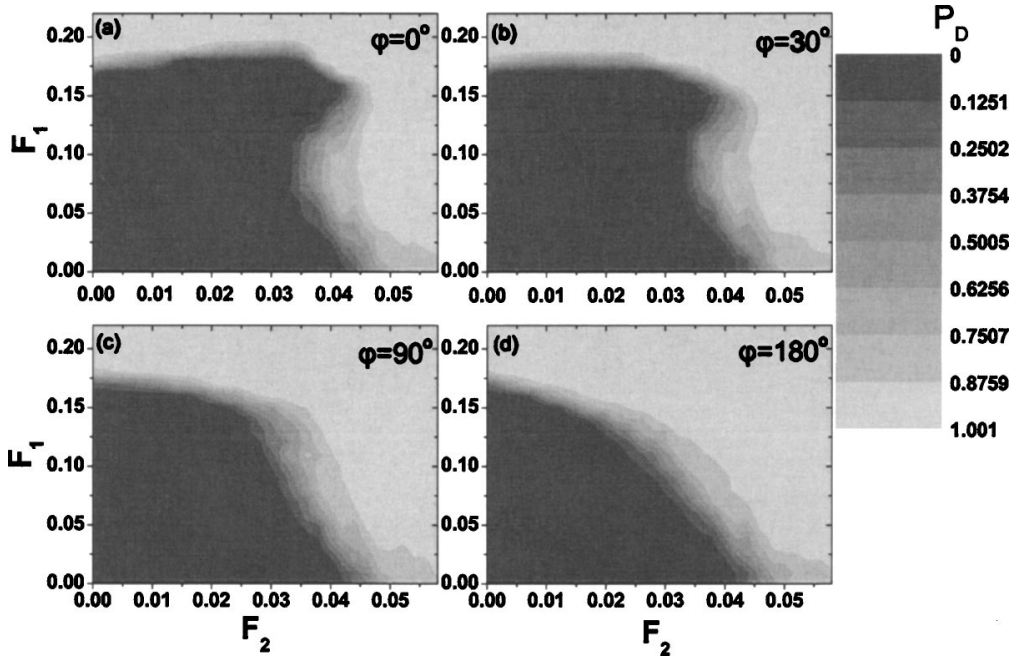

FIG. 2. Contour plots of dissociation probability $\mathrm{P}_{\mathrm{D}}$ in the plane $\mathrm{F}_{2}-\mathrm{F}_{1}$ for various relative phases: $\varphi=0^{\circ}$ (a), $\varphi=30^{\circ}$ (b), $\varphi=90^{\circ}$ (c), and $\varphi=180^{\circ}$ (d). For $\varphi=0$ notice the reduction of $P_{D}$ (stabilization) as $F_{2}\left(F_{1}\right)$ increases with $F_{1}\left(F_{2}\right)$ just above (below) the single laser dissociation threshold, as well as its association with the maximum sensitivity region.

tial increase in $P_{\mathrm{D}}$ and for relatively large slow laser amplitudes. Both stabilizations are $\varphi$ dependent and vanish for $\varphi$ $>90^{\circ}$.

The above calculations were performed using laser pulses of trapezoidal shape. The question that arises is whether and how the pulse shape and duration affect the $\varphi$ dependence and stabilization. We first look at the pulse duration. Figure 3(a) shows the dissociation sensitivity $\mathrm{SP}_{D}\left(F_{2}, F_{1}\right)$ for a pulse duration of 120 periods of the slow laser and a rising time of 30 periods. For this pulse, rising time is $25 \%$ of the total duration, as it is in the case of pulses of 40 periods that were used for the previous results. Comparison of this contour plot with that of Fig. 2 suggests that the impact of pulse duration on the effect caused by the relative phase is of minimal importance. However, the opposite conclusion is extracted for the impact of the variation in rising times, that is, for the impact of the pulse shape. Figure 3(b) depicts $\mathrm{SP}_{D}$ when long ac fields are considered. The difference with the previous $\mathrm{SP}_{D}$ contour plots is noticeable. The use of ac fields reduces the influence of $\varphi$ on the dissociation probability but leads to slightly larger $F_{1}-F_{2}$ regions where $\mathrm{SP}_{D}$ is greater than zero. Accordingly, it is found that the ac fields weaken both fast- and slow-laser stabilization phenomena.

\section{PHASE SPACE ORIGINS OF STABILIZATION AND OF RELATIVE PHASE EFFECTS}

When a nonlinear Hamiltonian oscillator is driven periodically, a series of nonlinear resonances consisting of alter- nating stable and unstable periodic orbits occurs at energies where the ratio of the frequency of the unperturbed oscillator $\Omega(\mathrm{E})$ and the driving frequency $\omega_{1}$ is rational, i.e.,

$$
\mathrm{m}_{1} \Omega(\mathrm{E})=\mathrm{n}_{1} \omega_{1},
$$

with $\mathrm{m}_{1}, \mathrm{n}_{1} \in \mathrm{Z}^{+}$.

For $\mathrm{n}_{1}=1$, there appear integral resonances, which typically dominate in phase space, while the smaller fractional resonances occur for $\mathrm{n}_{1} \neq 1$. The nonlinear resonances are intimately related to the occurrence of chaotic behavior in driven systems, since deterministic chaos arises first near the separatrices of their unstable periodic orbits. ${ }^{25}$ As the driving increases, the resonance zones with their surrounding chaotic layers widen and finally overlap each other, giving rise to global (or widespread) chaos. In spatially open systems, such as those modeling ionization or dissociation, the onset of global chaos leads to escaping trajectories. The field intensity leading to this onset is in fact the threshold field for ionization or dissociation, and can be predicted by the resonance overlapping criterion of Chiricov which has been applied for both Coulomb and Morse potentials with more or less successful results. ${ }^{15,17,26}$

The addition of a second driving field with frequency $\omega_{2}$ induces an additional series of nonlinear resonances at energies satisfying the relationship

$$
\mathrm{m}_{2} \Omega(\mathrm{E})=\mathrm{n}_{2} \omega_{2}
$$

with $\mathrm{m}_{2}, \mathrm{n}_{2} \in \mathrm{Z}^{+}$.

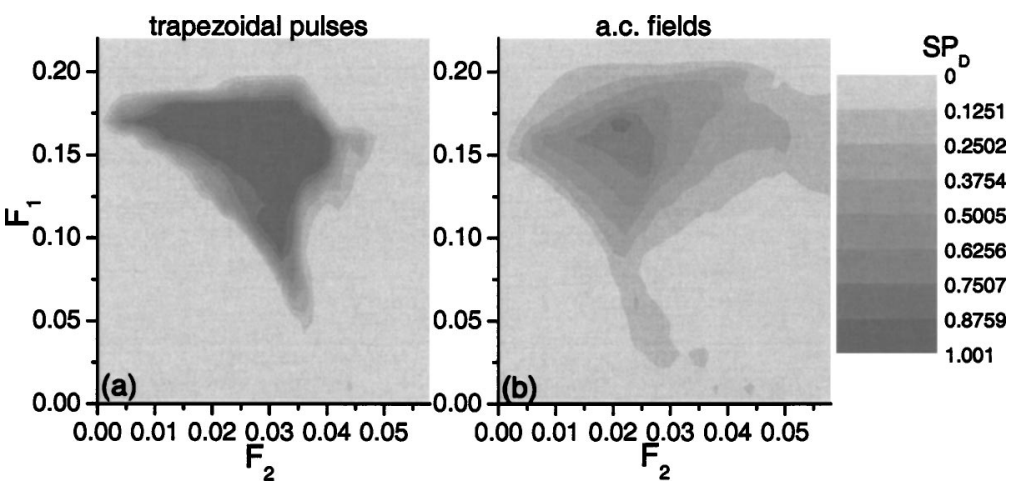

FIG. 3. Sensitivity $\mathrm{SP}_{\mathrm{D}}$ regions in the plane $\mathrm{F}_{2}-\mathrm{F}_{1}$ for a trapezoidal pulse, with duration and rising times four times longer than those used in previous figures (a) and for ac fields (b). We observe that the use of longer trapezoidal pulses with the same proportion of rising times does not affect the maximum sensitivity regions, whereas the use of ac fields weakens the relative phase affects and slightly enlarges the sensitivity regions. 

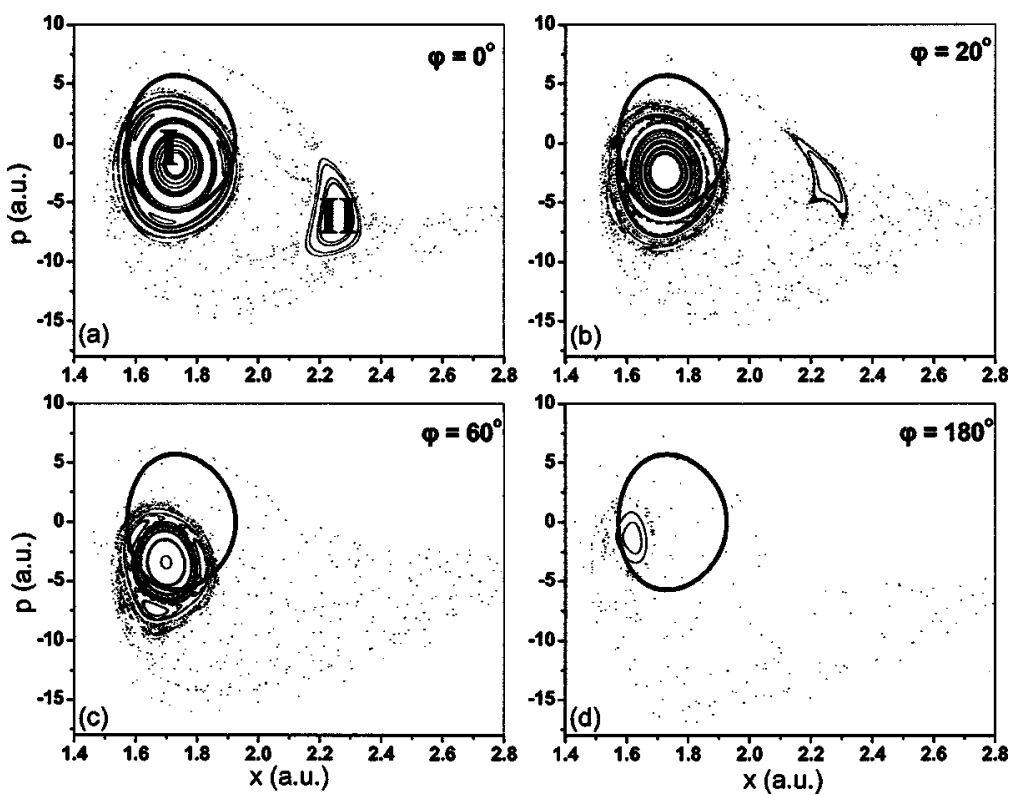

FIG. 4. Stroboscopic plots of phase space for $F_{1}$ $=0.18, \mathrm{~F}_{2}=0.02$, and $\varphi=0^{\circ}$ (a), $\varphi=20^{\circ}$ (b), $\varphi=60^{\circ}$ (c), and $\varphi=0^{\circ}(\mathrm{d})$. The regular region I contains remaining KAM tori of the unperturbed system, while the region II is the island of stability of the lowest-energy common resonance, corresponding to $\mathrm{n}_{1}=\mathrm{m}_{1}=1$ and $\mathrm{n}_{2}=1, \mathrm{~m}_{2}$ $=3$. The closed bold line represents in phase space the initial classical state whose overlap with stability regions gives roughly the nondissociating part of the state. Notice that the relative phase effect on $P_{D}$ is caused by the shrinking and slight movement of the KAM region I and the resulting decrease in its overlap with the initial state.
The energies at which either Eq. (8) or Eq. (9) hold give the so-called independent resonances that belong to either the first or the second resonance series. However, when the frequencies of the two driving fields are commensurate, there is the possibility for energies satisfying both Eqs. (8) and (9), i.e., $\Omega(E)=\left(n_{1} / m_{1}\right) \omega_{1}=\left(n_{2} / m_{2}\right) \omega_{2}$, and giving rise to common resonances. The existence of integral common resonances in dichromatically driven Hydrogen atoms was first noticed by Howard. ${ }^{13}$ However, no further investigation of their behavior was reported. Their principal role in the ionization process and its dependence on the relative field phase was elucidated by Sirko and $\mathrm{Koch}^{6}$ through a combined experimental-theoretical study of the microwave ionization of highly excited Hydrogen atoms. They showed that the experimentally revealed effects of $\varphi$ on the slow field threshold intensity for the onset of ionization can be interpreted by the phase space modifications caused by the variation of $\varphi$. In particular, they demonstrated that the relative phase can control the width of the common resonances in phase space and consequently their overlapping behavior and the associated ionization threshold field intensity. Their study focused on the behavior of the fractional common resonances of high order located near the initial excited state of their experiment.

In this work, the driving field is laser rather than microwave radiation and the potential is the Morse rather than the Coulomb. Furthermore, as initial conditions we choose the diatomic molecule to vibrate in its ground state. This means that the common resonances involved in dissociation dynamics are expected to be of the lowest order. For the chosen parameters (see Sec. III) the common resonance with the lowest energy $(E=0.29)$ corresponds to $n_{1}=m_{1}=1$ and $n_{2}$ $=1, \mathrm{~m}_{2}=3$. Furthermore, due to the low initial energy, the remaining KAM tori from the unperturbed system may also play an important role in dissociation dynamics.

Figure 4(a) shows a typical stroboscopic plot of phase space, which records the trajectories of the initial state at integral multiples of the period $\mathrm{T}_{1}$ of the slow laser. As we can see, there are two regular regions (I and II) inside a chaotic sea of dissociating trajectories. The regular region I consists of KAM tori of the unperturbed Morse potential that have survived in the strongly perturbed system of Fig. 4(a). They surround the stable periodic orbit emanating from the lowest energy fixed point of the Morse potential. On the other hand, the regular region II is in fact the island of stability corresponding to the lowest energy common resonance mentioned above $(E=0.29)$. The bold line shown also in Fig. 4(a) represents the ensemble of the initial conditions with energy $\mathrm{E}=\mathrm{E}_{0}=0.045$ and equidistributed angles $\theta$, as defined in Sec. II. The classical dissociation probability of the ground state is proportional to the part of the bold line lying in the chaotic sea.

The stroboscopic plot of Fig. 4(a) was taken for $\varphi=0$ and $F_{1}=0.18, F_{2}=0.02$, which belong to the region of maximum $\mathrm{SP}_{\mathrm{D}}$ (see Fig. 1). The remaining portions of Fig. 4 show the same part of the phase space for different relative phases: $\varphi=20^{\circ}$ (b), $\varphi=60^{\circ}$ (c), and $\varphi=180^{\circ}$ (d). The increase in $\varphi$ modifies both regular regions with more pronounced effect on the region II of the common resonance since it induces the disappearance of the resonance island for $\varphi$ $>25^{\circ}$. More detailed recording of the phase space structure as $\varphi$ increases indicates that this disappearance occurs gradually through a bifurcation process of the stable periodic orbit of the specific common resonance.

Although of paramount importance for the phase space topology, the disappearance of the resonance does not seem to affect significantly the dissociation probability, since there is no overlap of the island with the initial state. On the contrary, it is expected to influence the dissociation rate as it takes place in the dissociation paths of the escaping trajectories.

Less impressive but more influential on the dissociation dynamics is the effect of the variation of $\varphi$ on the KAM regular region I. As one can see in Fig. 4, increasing $\varphi$ causes the shrinking of that region and at the same time the movement of its position in phase space. The combination of these effects has drastic results concerning the overlapping of the KAM region with the line of the initial state and, in fact, 

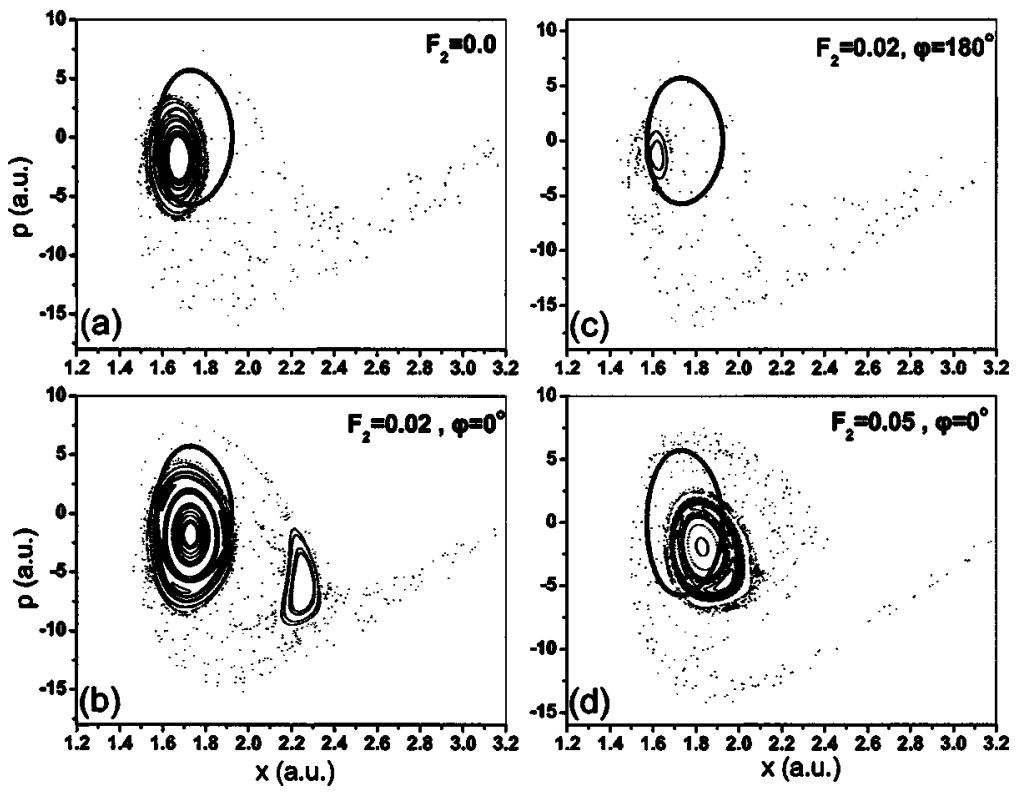

FIG. 5. Stroboscopic plots revealing the phase space origins of fast-laser stabilization effect. For all plots, $\mathrm{F}_{1}=0.18$, i.e., just above the single laser dissociation threshold, while the values of the second laser intensity and the relative phase are shown on the plots. The closed bold line represents, again, the ensemble of initial conditions.

constitutes the phase space origin of the relative phase effect on the dissociation probability in maximum sensitivity regions of the plane $F_{1}-F_{2}$. No such modifications of the KAM region $I$ have been observed for $F_{1}$ and $F_{2}$ outside maximum sensitivity regions.

In the preceding paragraph, we demonstrated numerically the possibility of both slow- and fast-laser stabilization of the dissociation dynamics when appropriate choices for the other laser field are made. Further, these stabilization effects are $\varphi$ sensitive; they are present for $\varphi=0^{\circ}$ but diminish as $\varphi$ increases. Here we show that, in phase space terms, this stabilization behavior is generated by the response of the KAM region I to laser intensity variations, in a similar way to the relative phase effects discussed above.

Let us first examine the phase space modifications in the fast laser stabilization. The slow laser intensity is set at $F_{1}$ $=0.18$ (see Fig. 5) and the stroboscopic plot with $\mathrm{F}_{2}=0$ is shown in Fig. 5(a). The resonance island has already disappeared and the only stability region in phase space is the remaining KAM tori. In Figs. 3(b) and 3(d) the evolution of the phase space structure for $\varphi=0^{\circ}$ as $F_{2}$ increases is monitored. For $\mathrm{F}_{2}=0.02$, [Fig. 3(b)], the common resonance island has emerged. At the same time, the KAM region is enlarged and has moved, leading to more overlapping with the KAM region of the initial state (depicted with the bold line) and hence to smaller dissociation probability.

Thus, the changes in phase space that occurred for constant $\mathrm{F}_{1}, \mathrm{~F}_{2}$ in the maximum sensitivity region and for decreasing $\varphi$ are also observed for constant $\mathrm{F}_{1}, \varphi=0^{\circ}$ and increasing $\mathrm{F}_{2}$. When $\mathrm{F}_{2}$ increases further, the resonance island disappears and the size of the KAM region begins to reduce with complete disappearance at $\mathrm{F}_{2} \sim 0.07$ [see Fig. 5(d)].

The situation changes drastically as $\varphi$ increases. Figure 5(c) exhibits the difference for $\varphi=180^{\circ}$. For $F_{2}=0.02$, no island of stability corresponding to the common resonance exists, while the size of the KAM region has obviously decreased. The monotonous reduction of the KAM area as $\mathrm{F}_{2}$ increases is the cause of the monotonous elevation of $\mathrm{P}_{\mathrm{D}}$ for $\varphi>90^{\circ}$ (see Fig. 2). Therefore, the response of the KAM region area to changes in $\mathrm{F}_{2}$ and its sensitivity to $\varphi$ can be used to interpret, in phase space terms, the form of the contour plots in Fig. 2 related to the fast stabilization phenomenon.

Similar phase space modifications seem to be behind the slow-laser stabilization effect. In this case, the stabilization takes place for quite large slow-laser intensities, $0.10<\mathrm{F}_{1}$ $<0.20$. In Fig. 6, we show the evolution of the phase space topology as $F_{1}$ varies in the stabilization region, while $F_{2}$ $=0.04$ and $\varphi=0^{\circ}$ [Figs. 6(a)-6(c)] or $\varphi=180^{\circ}$ Figs. $6(d)-6(f)$. We report again for $\varphi=0^{\circ}$ the apparent magnification of both KAM and common resonance regular regions as $\mathrm{F}_{1}$ increases, [see Fig. 6(b)], and the concomitant decrease in the overlapping of the initial state with the chaotic sea. Further increase in $\mathrm{F}_{1}$ leads to gradual shrinking of both regions, as Fig. 6(c) for $F_{1}=0.20$ indicates. Figures $6(d)$ and 6(e) reveal the impressive differentiation that the change in $\varphi$ brings about. For $\varphi=180^{\circ}$, the increase in $F_{1}$ from 0.10 to 0.15 results in reduction of the KAM region and total disappearance of the common resonance stability island. The dissociation probability $\mathrm{P}_{\mathrm{D}}$ increases monotonically, following the monotonous increase in the initial state overlapping with the chaotic sea. As $F_{1}$ grows up further, the KAM region disappears totally and for $\mathrm{F}_{1}=0.20$ the phase space is dominated by chaotic dissociating trajectories, as Fig. 6(f) shows.

\section{SUMMARY AND PERSPECTIVES}

In previous classical dynamics studies, ${ }^{11,12}$ we have shown that, in a single laser dissociation process, the redshift of the driving frequency giving the maximum dissociation probability can be explained in classical phase space terms by the relative movement and deformation of the KAM tori region and the lowest energy resonance island. Here, we explored the consequences of similar modification on a twolaser, dichromatically driven dissociation process, where the role of the important resonance is played by the lowest energy common resonance of two driving frequencies. In this case, the phase space modifications are induced by changing 

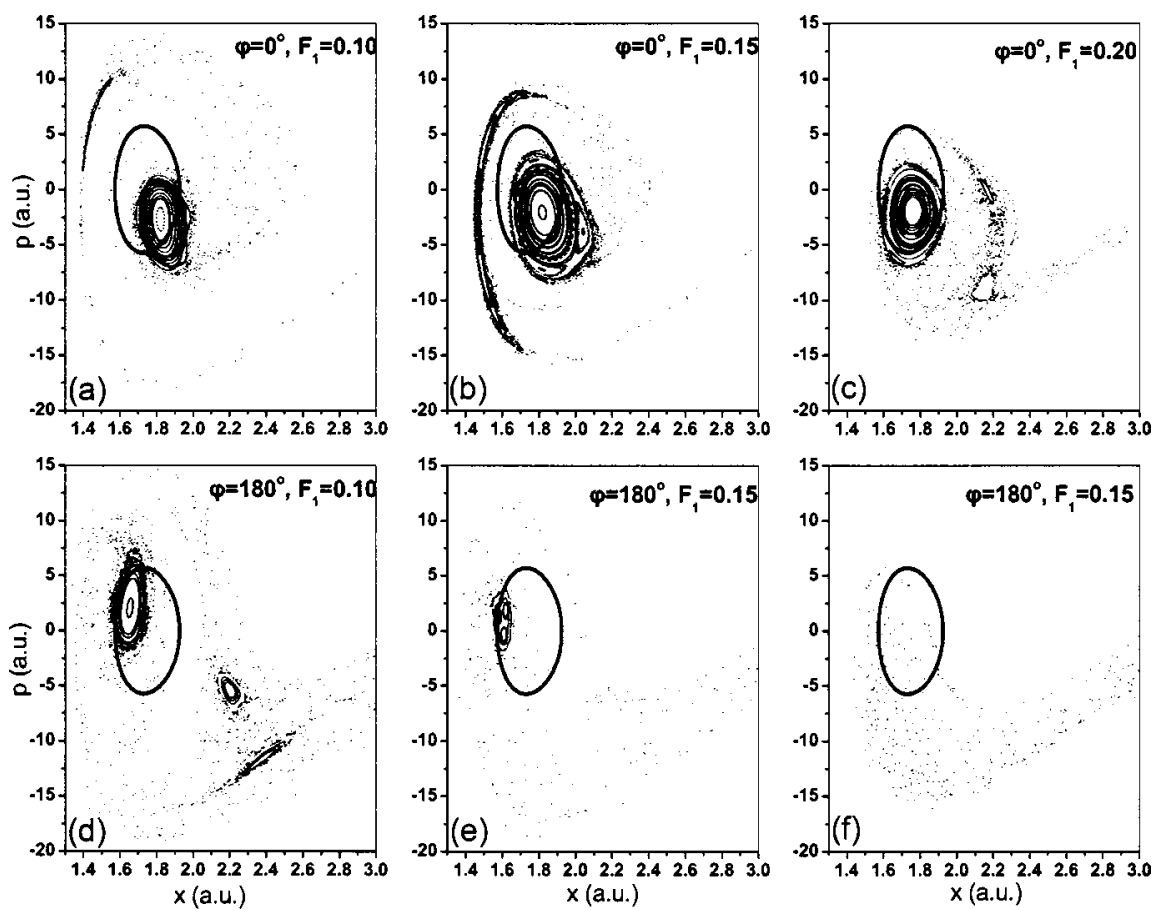

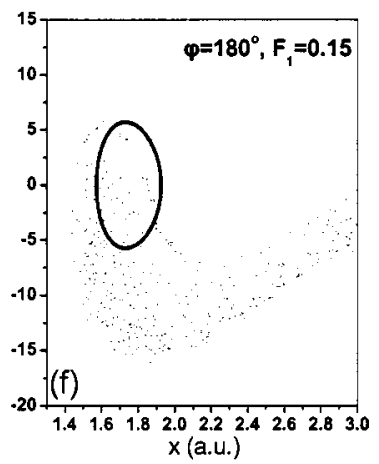

FIG. 6. Stroboscopic plots revealing the phase space origins of slow-laser stabilization effect. For all plots, $\mathrm{F}_{2}$ $=0.04$, i.e., just below the single laser dissociation threshold. The values of the other laser intensity and the relative phase are shown on the plots. The closed bold line represents the initial classical state. the relative phase $\varphi$ or one field intensity. Specifically, by carrying out extensive calculations, two different relative phase effects on the phase space structure of a dichromatically driven Morse oscillator have been determined. These add to the effect on the width of the common resonances, observed and discussed by Sirko and $\mathrm{Koch}^{9}$ in microwave ionization of highly excited Hydrogen.

The first of these effects is the deformation and movement of the KAM tori, and the second is the total disappearance of the common resonance island of stability. These effects occur for specific choices of the field intensities and are sensitive to the initial energy. The first of these is shown to be mostly responsible for the dependence of the dissociation probability on $\varphi$, since the line of initial conditions in phase space is partly overlapped by the KAM region in a way that is controlled by the relative phase. Furthermore, the addition of a second laser (irrelevant if it is faster or slower than the first) can cause similar phase space modification when the intensity of the first laser is chosen just above or below the single laser dissociation threshold and the relative field phase is kept sufficiently smaller than $\sim 90^{\circ}$. The enlargement of the KAM region in this case results in a suppression of the dissociation probability (stabilization) with the proper increase of the second laser intensity. We have found both slow- and fast-laser stabilization, depending on which laser controls the stabilization phenomenon. Increasing $\varphi$ gradually diminishes stabilization and raises dissociation probability.

Furthermore, we showed that the use of relatively short laser pulses enhances the stabilization $\varphi$-dependence effects, in comparison with ac fields, although the latter enlarges the regions in the intensity plane $\mathrm{F}_{1}-\mathrm{F}_{2}$ with $\mathrm{SP}_{\mathrm{D}}>0$. The destructive influence that the relative phase and the addition of the second laser have on the lowest energy common resonance does not affect directly the dissociation probability, since for the chosen parameters there is no overlap with the line of initial conditions. However, this is expected to affect the dissociation rate due to the stickiness effects always accompanying the presence of any island of stability. ${ }^{27,28}$ This is an issue for future work.

Additional open questions concern the relative phase and stabilization effects for other initial vibrational energies and different ratio of two applied frequencies. Also of interest is the possibility of drawing conclusions via the application of both analytical and numerical methods, on the different phase space structures that the dichromatic field generates, such as nested separatrices and $\varphi$-induced saddle node bifurcations.

Finally, the present classical mechanics study and its results open the possibility of further examination of the issue of correspondence with analogous quantum mechanical calculations of multiphoton dissociation.

\section{ACKNOWLEDGMENT}

This work was supported by the research program "Thalis" of the National Technical University of Athens.

${ }^{1}$ M. Shapiro and P. Brumer, Rep. Prog. Phys. 66, 859 (2003).

${ }^{2}$ F. Ehlotzky, Phys. Rep. 345, 175 (2001).

${ }^{3}$ R. J. Gordon, L. Zhu, and T. Seideman, Acc. Chem. Res. 32, 1007 (1999). ${ }^{4}$ D. W. Schumacher, F. Weihe, H. G. Muller, and P. H. Bucksbaum, Phys. Rev. Lett. 73, 1344 (1994).

${ }^{5}$ L. Sirko, S. A. Zelazny, and P. M. Koch, Phys. Rev. Lett. 87, 043002 (2001).

${ }^{6}$ L. Sirko and P. M. Koch, Phys. Rev. Lett. 89, 274101 (2002).

${ }^{7}$ K. J. Schafer and K. C. Kulander, Phys. Rev. A 45, 8026 (1992).

${ }^{8}$ T. W. Cheng, J. Liu, and S. Chen, Phys. Rev. A 59, 1451 (1999).

${ }^{9}$ Th. Mercouris and C. A. Nicolaides, Phys. Rev. A 63, 013411 (2001).

${ }^{10}$ E. Charron, A. Giusti-Suzor, and F. H. Mies, Phys. Rev. A 49, R641 (1994).

${ }^{11}$ V. Constantoudis and C. A. Nicolaides, Phys. Rev. E 64, 046204 (2001).

${ }^{12}$ V. Constantoudis and C. A. Nicolaides, Phys. Rev. A 55, 1325 (1997).

${ }^{13}$ J. E. Howard, Phys. Lett. A 156, 286 (1991).

${ }^{14}$ J. R. Stine and D. W. Noid, Opt. Commun. 31, 161 (1979).

${ }^{15}$ M. E. Goggin and P. W. Milonni, Phys. Rev. A 37, 796 (1988). 
${ }^{16}$ A. Guldberg and G. D. Billing, Chem. Phys. Lett. 186, 229 (1991).

${ }^{17}$ B. Wu and W-K. Liu, Physica A 205, 470 (1994).

${ }^{18}$ M. E. Goggin and P. W. Milonni, Phys. Rev. A 38, 5174 (1988).

${ }^{19}$ Y. Gu and J-M. Yuan, Phys. Rev. A 36, 3788 (1987).

${ }^{20}$ M. Thachuk and D. M. Wardlaw, J. Chem. Phys. 102, 7462 (1995).

${ }^{21}$ C. A. Nicolaides, Th. Mercouris, and I. D. Petsalakis, Chem. Phys. Lett 212, 685 (1993).

${ }^{22}$ P. C. Dardi and S. K. Gray, J. Chem. Phys. 77, 1345 (1982).
${ }^{23}$ R. Heather and H. Metiu, J. Chem. Phys. 88, 5496 (1988).

${ }^{24}$ P. M. Koch, S. A. Zelazny, and L. Sirko, J. Phys. B 36, 4755 (2002).

${ }^{25}$ A. J. Lichtenberg and M. A. Lieberman, Regular and Chaotic Dynamics (Springer, New York, 1992).

${ }^{26}$ R. V. Jensen, Phys. Rev. A 30, 386 (1984).

${ }^{27}$ C. Efthimiopoulos, G. Contopoulos, N. Voglis, and R. Dvorak, J. Phys. A 30, 8167 (1997)

${ }^{28}$ G. M. Zaslavsky, Phys. Rep. 371, 461 (2002). 